\title{
Designing Cinematic Lighting by Relighting in MR-based Pre-visualization
}

Ryosuke Ichikari* Ryohei Hatano

\author{
Toshikazu Ohshima \\ Ritsumeikan University
}

Fumihisa Shibata

Hideyuki Tamura

\begin{abstract}
This paper describes a relighting method of designing cinematic lighting for filmmaking. The relighting method enables mixed reality based pre-visualization called MR-PreViz to change conditions of illumination. The method allows the MR-PreViz to have additional virtual lighting and the removal of actual illumination in designing cinematic lighting. The effects of lighting are applied correctly to both real objects and virtual objects.
\end{abstract}

CR Categories: H.5.1 [Multimedia Information Systems]: Artificial, augmented, and virtual realities;

Keywords: Mixed Reality, Pre-visualization, Relighting

\section{Introduction}

We are developing a previsualization method called MR-PreViz, which utilizes mixed reality technology for filmmaking[Tenmoku et al. 2006]. To expand the ability to visualize final image of the movie using MR-PreViz, we focus on the "look" which refers to the feeling of an image provided by lighting and color tone. In this paper, we introduce a relighting method for MR-PreViz movies for visualizing "look". The relighting method allows the MR-PreViz movies to have additional virtual lighting and the removal of actual illumination. The conventional relighting methods have realized relighting only indoors. Our method adopts simple and approximative approach to realize indoor-outdoor relighting.

\section{Workflow of Relighting}

The purpose of our method is to visualize the "look" before the time of shooting not rigorous optical simulation of lighting is not required. Therefore the method adopts approximative approach for indoor-outdoor relighting as following and shown in Figure 1.

Step 1 Shadow removal

The proposed method removes shadows from the input images as much as possible to avoid an optical paradox between real shadows and virtual shadows.

Step 2 Adjusting color tone

After shadow removal, the color tone is adjusted by multiplying arbitrary values with respect to each color channel. As a result of this process, we can change an image of daylight into an image of a night scene. This process approximates environmental light.

Step 3 Adding lighting effects to MR-PreViz images

After color correction, real and virtual objects in the MRPreViz images are illuminated by virtual lighting. To optically correctly illuminate objects, we estimate reflectance properties of the real objects.

\section{Removal of Shadow}

To remove shadow, distributions of pixel color in the shadow area are conformed to one in a sunny area. Therefore, manual assign-

\footnotetext{
*e-mail: ichikari@rm.is.ritsumei.ac.jp
}
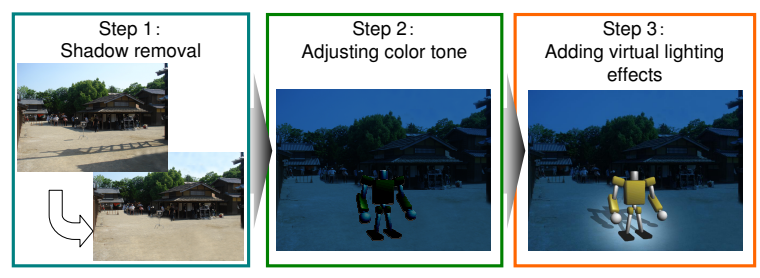

Figure 1: Flow of our relighting method.
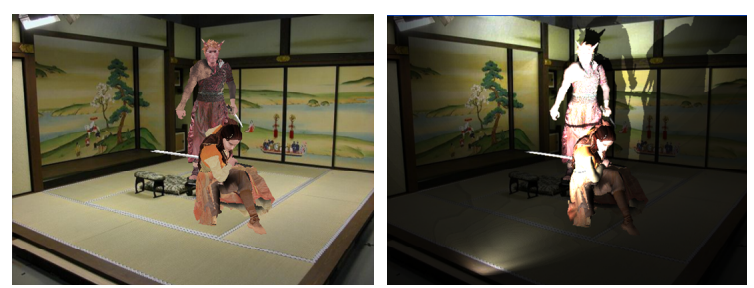

Figure 2: Result of relighting.(left:input, right:result)

ments of these areas at reference frames are required. The proposed method provides the ability to remove soft shadows whose silhouettes are blurred. Soft shadows are often observed in actual scenes. Nonetheless, there is no dominant method for removing them. To remove soft shadow, we propose the use of a shadow gradation map (SGM) which indicates density of shadow. The amount of color adjustment required is determined according to the density of shadow.

\section{Virtual lighting to MR-PreViz}

Virtualization of the real objects on the shooting site is required to illuminate them optically correctly. In this method, we estimate rough geometry and reflectance property of the illuminated surface. We obtain the relationship between pixel RGB value and illuminance on the surface to approximate the reflectance property. After the virtualization, MR-PreViz images are illuminated by virtual lighting. To illuminate them in the same way as with a real lamp, we use IES data, which is the light distribution information of the lamp. The amount of increased illuminance, by the lighting, is calculated for each pixel on the illuminated surface, according to distance from the lamp and the angle of the light axis. As a result shown in Figure 2, the "look" of the scene is successfully designed. We have applied the method to whole MR-PreViz movies using the camera-work data at the MR-PreViz shooting.

\section{Acknowledgements}

This research is partly supported by the CREST Program of JST.

\section{References}

Tenmoku, R., Ichikari, R., Shibata, F., Kimura, A., And TAMuRA, H. 2006. Design and prototype implementation of $\mathrm{mr}$ pre-visualization workflow. In DVD-ROM Proc. of the Int'l Workshop on Mixed Reality Technology for Filmmaking, 1-7. 